\title{
Is Amplitude Loss of Sonic Waveforms Due to Intrinsic Attenuation or Source Coupling to the Medium?
}

Scientific Investigations Report 2006-5120 


\section{Is Amplitude Loss of Sonic Waveforms Due to Intrinsic Attenuation or Source Coupling to the Medium?}

By Myung W. Lee

Scientific Investigations Report 2006-5120 


\section{U.S. Department of the Interior \\ Dirk Kempthorne, Secretary \\ U.S. Geological Survey \\ P. Patrick Leahy, Acting Director}

\section{U.S. Geological Survey, Reston, Virginia: 2006}

Posted online June 2006

Version 1.0

This publication is available only online at:

World Wide Web: http://www.usgs.gov/pubprod

Telephone: 1-888-ASK-USGS

For more information on the USGS - the Federal source for science about the Earth, its natural and living resources, natural hazards, and the environment:

World Wide Web: http://www.usgs.gov

Telephone: 1-888-ASK-USGS

Any use of trade, product, or firm names is for descriptive purposes only and does not imply endorsement by the U.S. Government.

Although this report is in the public domain, permission must be secured from the individual copyright owners to reproduce any copyrighted materials contained within this report.

Suggested citation:

Lee, M.W., 2006, Is amplitude loss of sonic waveforms due to intrinsic attenuation or source coupling to the medium?: U.S. Geological Survey Scientific Investigations Report 2006-5120, 13 p. 


\section{Contents}

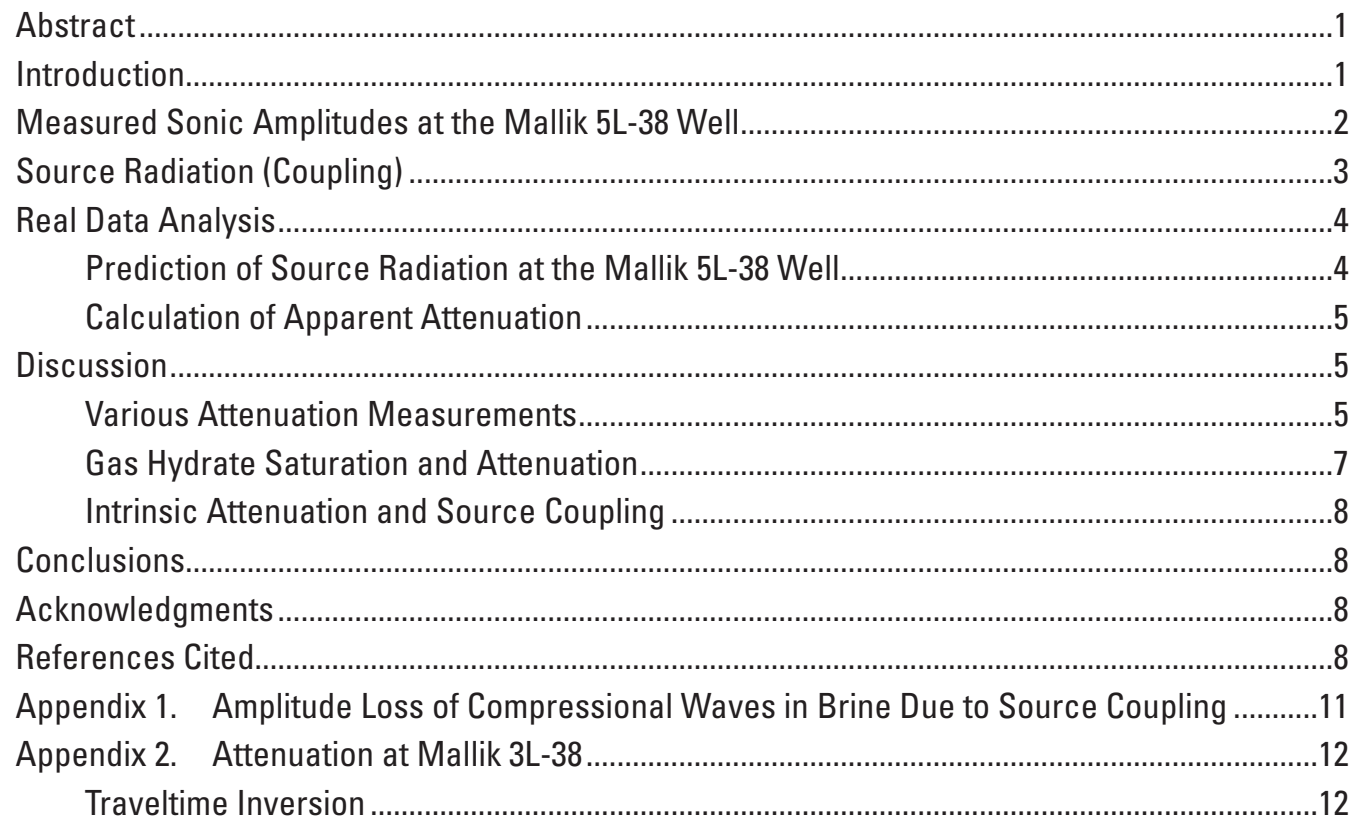

\section{Figures}

1. Measured sonic and Stoneley-wave amplitudes at Mallik 5L-38 well, Mackenzie Delta, Northwest Territories, Canada ..........................................................

2. Normalized amplitudes of P-waves, S-waves, and Stoneley waves..................................

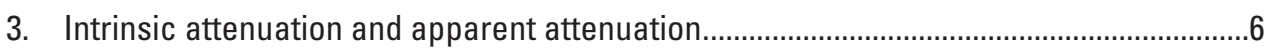

\section{Appendix Figures}

1-1. P-wave amplitude loss due to source coupling compared to amplitude loss

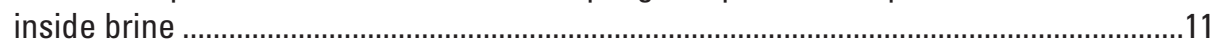

2-1. Spectral ratios at Mallik 3L-58 well ..............................................................................12

2-2. Velocities derived from VSP data at Mallik $3 L-58$ well and well-log velocities measured at Mallik 5L-38 


\section{Conversion Factors}

\begin{tabular}{lcl}
\hline Multiply & By & To obtain \\
\hline meter $(\mathrm{m})$ & Length & \\
kilometer $(\mathrm{km})$ & 3.281 & foot $(\mathrm{ft})$ \\
Multiply & 0.6214 & mile $(\mathrm{mi})$ \\
\hline & By & To obtain \\
\hline foot (ft) & Length & \\
mile (mi) & 0.3048 & meter $(\mathrm{m})$ \\
\hline
\end{tabular}




\title{
Is Amplitude Loss of Sonic Waveforms Due to Intrinsic Attenuation or Source Coupling to the Medium?
}

\author{
By Myung W. Lee
}

\begin{abstract}
Sonic waveforms acquired in gas-hydrate-bearing sediments indicate strong amplitude loss associated with an increase in sonic velocity. Because the gas hydrate increases sonic velocities, the amplitude loss has been interpreted as due to intrinsic attenuation caused by the gas hydrate in the pore space, which apparently contradicts conventional wave propagation theory. For a sonic source in a fluid-filled borehole, the signal amplitude transmitted into the formation depends on the physical properties of the formation, including any pore contents, in the immediate vicinity of the source. A signal in acoustically fast material, such as gashydrate-bearing sediments, has a smaller amplitude than a signal in acoustically slower material. Therefore, it is reasonable to interpret the amplitude loss in the gas-hydrate-bearing sediments in terms of source coupling to the surrounding medium as well as intrinsic attenuation. An analysis of sonic waveforms measured at the Mallik 5L-38 well, Northwest Territories, Canada, indicates that a significant part of the sonic waveform's amplitude loss is due to a source-coupling effect. All amplitude analyses of sonic waveforms should include the effect of source coupling in order to accurately characterize the formation's intrinsic attenuation.
\end{abstract}

\section{Introduction}

In sediments, the presence of gas hydrate-a crystalline substance composed of a "cage" of water molecules that surround gas molecules - significantly changes the sediments' physical properties; for example, their elastic velocities are higher than the elastic velocities of sediments without gas hydrates. Various seismic survey methods utilize this velocity increase as a means of identifying and quantifying gas hydrate concentrations.

The amplitude loss of sonic waveforms, measured by a Dipole Shear Sonic Imager (DSI) (12 kHz for monopole source and $2 \mathrm{kHz}$ for dipole source), has been interpreted to be due to intrinsic attenuation. For example, (1) Guerin and Goldberg (2002) analyzed the DSI sonic waveforms measured at the Mallik 2L-38 well, Mackenzie Delta, Canada, and suggested that the $\mathrm{P}$ - and $\mathrm{S}$-wave attenuation increases as the gas hydrate saturation increases. (2) Later, Guerin and others (2005) applied the same analysis techniques to the sonic waveforms acquired at the Mallik 5L-38 well and thereby confirmed the validity of the previous analysis at the neighboring Mallik 2L-38 well. (3) In addition, Matsushima (2005a) arrived at the same conclusion as Guerin and others (2005) on the basis of an analysis of sonic waveforms acquired at the Nankai Trough exploratory well offshore of Tokai, central Japan.

Inversion of hole-to-hole seismic data acquired near the Mallik 5L-38 well site allowed Bauer and others (2005) to conclude that the amplitude loss in the frequency range of 200-2,000 Hz increases as the gas hydrate saturation increases, but the magnitude of $\mathrm{P}$-wave attenuation is about one-half of that determined by the sonic waveform analysis (Guerin and others, 2005). Bauer and others attributed these $\mathrm{P}$-wave data to frequency-dependent attenuation, whereby the amplitude loss increases with increasing frequency.

Several investigators proposed various mechanisms to explain the peculiarly large amplitude loss inferred from the sonic waveform analysis of gas-hydrate-bearing sediments (GHBS) and from the hole-to-hole seismic data. These investigators then applied the amplitude loss as a parameter to quantify the amount of gas hydrate (Guerin and Goldberg, 2002; Chand and Minshull, 2004; Dvorkin and Uden, 2004; Guerin and others, 2005).

However, the present study uses surface vertical seismic profile (VSP) data to demonstrate that the amplitude loss of sonic waveforms measured for GHBS is less than that measured in water-saturated sediments. Rossi and others (2005) used tomographic analysis of three-component seismic data from an ocean-bottom seismograph (OBS) array offshore northwest Svalbard to conclude that GHBS manifest themselves with high $\mathrm{P}$-wave velocities and low $\mathrm{P}$-wave attenuations, in agreement with the analysis discussed in this report (see appendix 2). Gei and Carcione (2003) suggested that attenuation should decrease with increasing gas hydrate saturation, and they argued that gas hydrate makes sediments more cohesive.

Thus, two opposing views and contrasting analyses exist for the amplitude loss of sonic waveforms measured for GHBS. Attenuations estimated at low frequency in sediments with various amounts of gas hydrate (in surface VSP data and 
OBS data) suggest that gas hydrate presence in sediments decreases their intrinsic attenuation. However, attenuations estimated at higher frequencies (in sonic and hole-to-hole seismic data) suggest that gas hydrate presence in sediments increases their intrinsic attenuation. These different views have been based on data acquired with different source environments as well as different input frequencies.

Theories predict that the strength of radiated energy strongly depends on the elastic parameters of the surrounding medium. For example, the displacement from a given point source acting on a half space decreases as the density and elastic velocities of the half space increase. The source environment did not change during the acquisition of VSP and OBS data, but continuously changed during the acquisition of sonic and hole-to-hole seismic data as the sources were located at different depths. Therefore, the measured amplitude loss of sonic waveforms could have been the effect of the source coupling to the surrounding medium as well as changing the intrinsic attenuation.

The seismic responses of borehole sources (monopole and dipole) inside a fluid-filled hole can be calculated by using numerical methods (for example, Tsang and Rader, 1979; Cheng and Toksöz, 1981; Kurkjian and Chang, 1986). However, in the present report, closed-form solutions under the assumption of far-field and low-frequency approximation of borehole sources by Lee and Balch (1982) and Lee (1986) are used to explain the amplitude loss observed in sonic waveforms at the Mallik 5L-38 well.

\section{Measured Sonic Amplitudes at the Mallik 5L-38 Well}

Sonic waveforms were measured by the DSI tool, and examples of monopole and dipole waveforms are shown in figure 1, modified from Guerin and others (2005). For comparison, the Stoneley-wave amplitude is shown (fig. 1) with a fullbore formation microimager (FMI) log, modified from Collett and others (2005). Zones with dark color in the FMI image represent low-resistivity zones, which correspond to little, if any, gas hydrate, whereas zones with light color indicate zones highly saturated in gas hydrate.

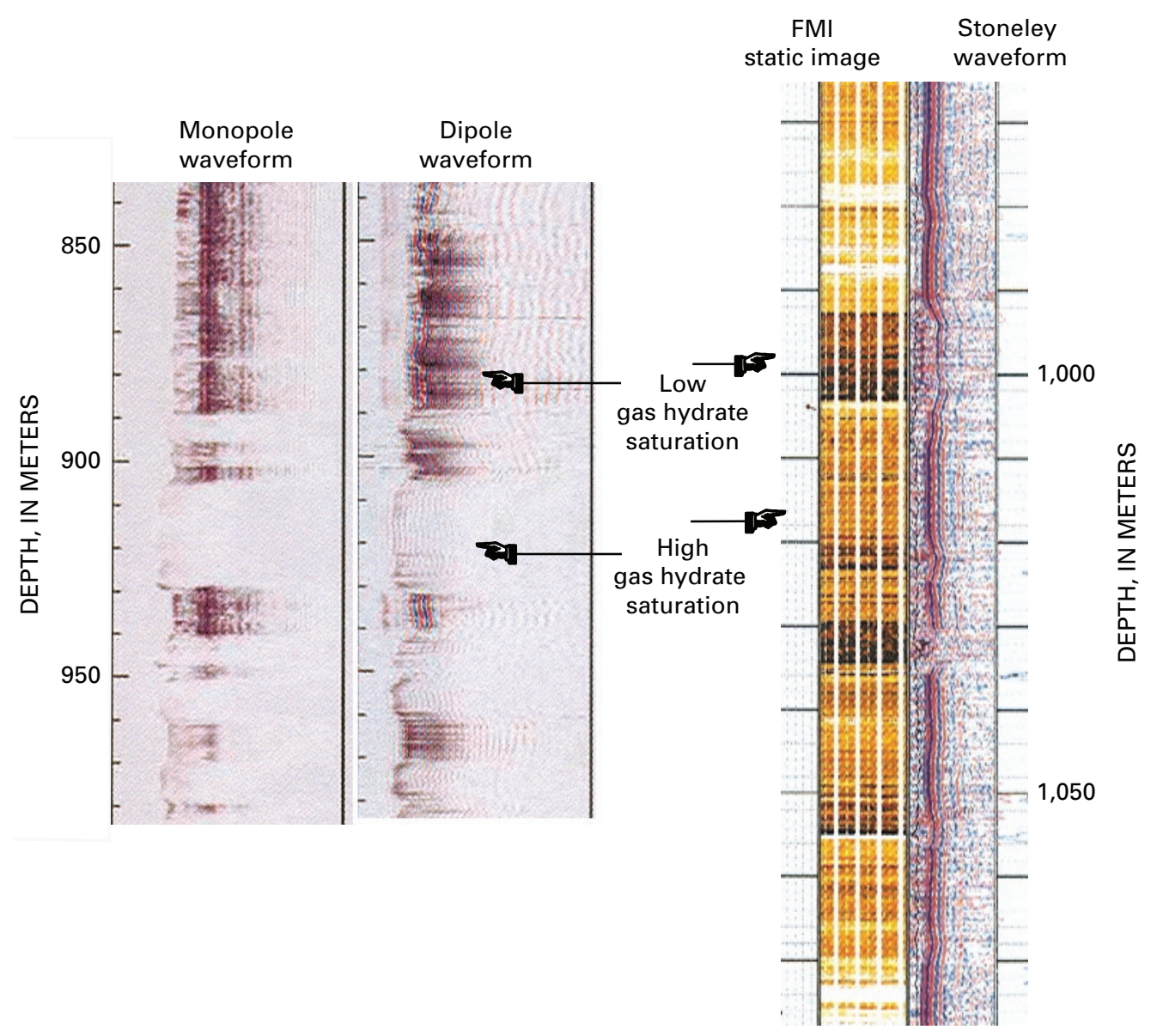

Figure 1. Measured sonic and Stoneley-wave amplitudes at the Mallik 5L-38 well, Mackenzie Delta, Northwest Territories, Canada. The monopole and dipole waveforms are modified from Guerin and others (2005). The Stoneley-wave amplitude and borehole formation microimager (FMI) static image are modified from Collett and others (2005). 
In the depth intervals 890-895 m, 910-930 m, and 950-960 m, which are highly saturated in gas hydrate, the monopole and dipole amplitudes are significantly reduced compared to the amplitudes in other intervals (Collett and others, 2005). Based on the sonic waveforms, shown in figure 1 , and gas hydrate saturations estimated from the nuclear magnetic resonance (NMR) tool (Kleinberg and others, 2005), the following observations can be made:

1. Both monopole and dipole waveform amplitudes are much lower in the intervals that are highly saturated in gas hydrate.

2. The monopole amplitude loss appears to be slightly greater than the dipole amplitude loss.

3. The Stoneley-wave amplitude is higher in intervals highly saturated with gas hydrate than in intervals with little or no gas hydrate.

Guerin and Goldberg (2002) attributed some energy loss to the greater velocity contrast between the borehole fluid and the formation, yet their estimated intrinsic attenuation has to be appreciably higher in GHBS than in non-gas-hydratebearing sediments (NGHBS) to model the amplitude loss observed at the Mallik 2L-38 well. They also noted that the intrinsic attenuation increase suppresses later arrivals such as the Stoneley mode in dipole waveforms. This amplitude prediction for the Stoneley wave by Guerin and Goldberg (2002) is opposite to the results shown by Collett and others (2005) in the Mallik 5L-38 well (fig. 1). Because the propagation mode of the Stoneley wave is different from that of the monopole Pwave, it is also questionable to attribute amplitude loss of the Stoneley wave to the intrinsic attenuation of the formation.

This report attempts to explain the amplitude variations of P-waves, S-waves, and Stoneley waves for GHBS (as shown in fig. 1) by using source coupling to the surrounding medium rather than by using intrinsic attenuation.

\section{Source Radiation (Coupling)}

In order to semiquantitatively analyze sonic log amplitudes, the radiation pattern valid under the assumption of the low-frequency and far-field approximation derived by Lee and Balch (1982) and Lee (1986) is used. The responses of various waves in real data obtained from monopole and dipole sources are more complicated than those indicated in this report. However, this simple approach can be used to predict a significant part of the measured amplitude loss, as shown later.

A brief theoretical analysis appropriate for this investigation follows. Let subscript 1 denote the borehole fluid and subscript 2 denote the surrounding solid; $\alpha, \beta$, and $\rho$ represent the P-wave velocity, $\mathrm{S}$-wave velocity, and density, respectively; $R$ is the distance from the source to the receiver, and $\omega$ is the frequency. In a spherical coordinate system $(R, \theta, \phi)$, a radial displacement field $\left(U_{\mathrm{r}}\right)$ due to an initial displacement source $\left(V_{0}\right)$ that acts on the axis of a fluid-filled borehole is given by the following formula in the frequency domain (Lee and Balch, 1982):

$$
U_{\mathrm{r}}=\frac{i \omega V_{0} \rho_{1}}{4 \pi \rho_{2} \alpha_{2}} \times \frac{1-2 \beta_{2}^{2} \cos ^{2} \phi / \alpha_{2}^{2}}{T_{\alpha}+\rho_{1} / \rho_{2}} \times \frac{e^{-i k_{\alpha_{2}} R}}{R},
$$

where

$$
T_{\alpha}=\frac{\beta_{2}^{2}}{\alpha_{1}^{2}}\left(1-\frac{\alpha_{1}^{2} \cos ^{2} \phi}{\alpha_{2}^{2}}\right),
$$

$$
k_{\alpha_{2}}=\frac{\omega}{\alpha_{2}},
$$

and

$$
i=\sqrt{-1}
$$

When a symmetrical source (like a monopole source) is applied inside a fluid-filled borehole, tube waves (that is, the Stoneley-wave type) are generated and travel with a velocity of $C_{\mathrm{T}}$. Note that a tube wave is a boundary wave propagating along a fluid-filled borehole. The pressure response of a tube wave $\left(P_{\mathrm{T}}\right)$ is given by the following formula from Lee and Balch (1982):

$$
P_{\mathrm{T}}=\frac{i \omega V_{0} \rho_{1} C_{\mathrm{T}}}{2 \pi a^{2}} \propto C_{\mathrm{T}},
$$

where $a$ is the borehole radius and $C_{\mathrm{T}}$ is the tube-wave velocity, which is given by

$$
C_{\mathrm{T}}=\left(\frac{1}{\alpha_{1}^{2}}+\frac{\rho_{1}}{\rho_{2} \beta_{2}^{2}}\right)^{-1 / 2} \text {. }
$$

When a radial stress source with a constant magnitude $T_{\text {rs }}$ is applied to the wall of a fluid-filled borehole, the azimuthal component of displacement $\left(U_{\theta}\right)$ is written as follows (Lee, 1986):

$$
U_{\theta}=T_{\mathrm{rs}}\left[\sin \theta+\frac{i \omega a \sin 2 \theta \sin \phi}{\beta_{2}\left(1-\beta_{2}^{2} / \alpha_{2}^{2}\right)}\right] \frac{e^{-i k_{\beta_{2}} R}}{R},
$$

where

$$
T_{\mathrm{rs}}=\frac{a T_{0}}{4 \pi \rho_{2} \beta_{2}^{2}},
$$


and $k_{\beta_{2}}=\omega / \beta_{2}$.

The monopole response of the sonic tool is simulated by using equation 1 , and the amplitude response of the monopole $\left(U_{\mathrm{r}}\right)$ is given by equation 5 when $\phi=\pi / 2$ (perpendicular to the borehole):

$$
U_{\mathrm{r}} \propto \frac{1}{\rho_{2} \alpha_{2}\left(\beta_{2}^{2} / \alpha_{1}^{2}+\rho_{1} / \rho_{2}\right)} .
$$

A dipole source can be approximated by using two opposing radial sources $180^{\circ}$ apart in the azimuthal direction. From equation 4, the dipole response is given by

$$
U_{\theta} \propto \frac{1}{\rho_{2} \beta_{2}^{2}}
$$

The azimuthal variation for the acoustic field (the radiation pattern on a plane perpendicular to the borehole or radiation pattern at $\phi=\pi / 2$ ), as calculated from equation 1 for the monopole and equation 4 for the dipole, agrees with the radiation pattern for the multiple acoustic sources of the logging system (Tang and others, 2002).

The Stoneley waves are dispersive, so the velocity depends on the frequency. The tube wave is the low-frequency component of the Stoneley wave and is nondispersive as shown in equation 3. In this analysis, it is assumed that the pressure response of the tube wave given in equation 2 is a good approximation of the pressure response of the Stoneley wave.

The amplitude loss (that is, the attenuation) $\left(Q^{-1}\right)$ of each wave is derived from the following equation (Schön, 1998):

$$
Q^{-1}=\frac{V}{\pi f} \frac{1}{x_{2}-x_{1}} \log \left(\frac{A\left(x_{1}\right)}{A\left(x_{2}\right)}\right),
$$

where $V$ is the velocity, $f$ is the linear frequency, $A\left(x_{1}\right)$ is amplitude at location $x_{1}$, and $A\left(x_{2}\right)$ is the amplitude at location $x_{2}$. The amplitude at a distance $R$ is given by

$$
A \propto e^{-\alpha R}
$$

where $\alpha=\pi \phi Q^{-1 / V}$.

A relative amplitude loss due to source coupling is independent of the distance the wave traveled, whereas the amplitude loss due to intrinsic attenuation is dependent on the travel distance. In order to meaningfully compare amplitude loss due solely to source coupling with the measured attenuation, an apparent attenuation coefficient $\left(\alpha_{\mathrm{a}}\right)$ is defined as the attenuation that reduces the amplitude as much as the amplitude loss due to source coupling. In other words, the wave amplitude due to the coupling effect $\left(A_{c}\right)$ is defined as

$$
A_{\mathrm{c}} \propto e^{-\alpha_{\mathrm{a}} R} .
$$

If a constant excitation frequency and a constant strength of source are assumed, the apparent attenuation $\left(Q_{\mathrm{a}, \mathrm{j}}\right)$ due to source coupling can be written as

$$
Q_{\mathrm{a}, j}^{-1} \propto V_{j} \log \left(A_{\mathrm{c}, j}\right)
$$

Therefore,

$$
\frac{Q_{\mathrm{a}, j}^{-1}}{Q_{\mathrm{a}, 1}^{-1}}=\frac{V_{j} \log \left(A_{\mathrm{c}, j}\right)}{V_{1} \log \left(A_{\mathrm{c}, 1}\right)} .
$$

If amplitudes normalized by $A_{c, 1}$ are used, equation 10 can be written as

$$
Q_{\mathrm{a}, j}^{-1}=Q_{a, 1}^{-1} \frac{V_{j} \log \left(G_{j} C\right)}{V_{1} \log \left(G_{1} C\right)}=Q_{\mathrm{a}, 1}^{-1} \frac{V_{j} \log \left(G_{j} C\right)}{V_{1} \log C},
$$

where $G_{j}$ is the normalized amplitude and $C$ is a scalar to convert $G_{j}$ back to the true amplitude in equation 10 . Equation 11 indicates that if the apparent attenuation at $j=1$ is known, apparent attenuations corresponding to the source coupling can be evaluated with known $C$ and normalized amplitudes.

In order to estimate apparent attenuation, the scalar $C$ in equation 11 is computed through the use of the following equation:

$$
C=A_{\mathrm{c}, 1}=e^{-Q_{\mathrm{a}, 1}^{-1} f \pi R / V_{1}} .
$$

\section{Real Data Analysis}

\section{Prediction of Source Radiation at the Mallik 5L-38 Well}

Figure 2 shows the normalized amplitude responses of $\mathrm{P}$-waves, $\mathrm{S}$-waves, and tube waves calculated by using the geophysical logs at the Mallik 5L-38 well and the source radiations (or source coupling to the formation) given in equations 2, 5, and 6 for the Stoneley-wave, P-wave, and S-wave responses, respectively. Like sources, receivers would have receiver coupling to the surrounding medium. However, in all amplitude computations, the receiver coupling is ignored.

As shown in the observed amplitude variation, the source radiation predicts amplitude loss to be largest for the monopole (P-wave response) and smallest for the tube wave. However, the amplitude loss of the $S$-wave due to source coupling is as great as that for the $\mathrm{P}$-wave. As the $\mathrm{P}$-wave velocity (or $\mathrm{S}$-wave velocity) increases, the amplitude response of the $\mathrm{P}$ wave (or S-wave) decreases. The tube-wave velocity increases as the formation $\mathrm{S}$-wave velocity increases. Figure 2 indicates that the tube-wave amplitude is higher where the S-wave 


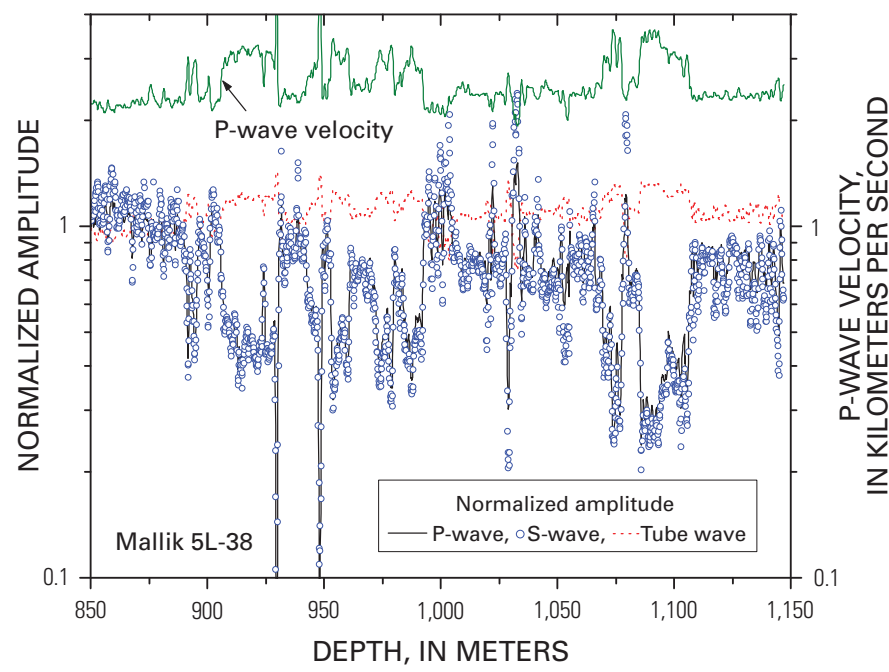

Figure 2. Normalized amplitudes of P-waves, S-waves, and Stoneley waves calculated by using the source coupling with measured P-wave velocities at the Mallik 5L-38 well. A monopole is simulated by using a symmetrical volume source, a dipole source is simulated by using two opposite radial sources $180^{\circ}$ apart, and the Stoneley wave is approximated by using the tube wave due to a symmetrical volume source.

velocity (the $\mathrm{S}$-wave velocity is proportional to the $\mathrm{P}$-wave velocity) is higher, in agreement with the measured amplitude variation of the Stoneley wave shown in figure 1 .

The details of the monopole and dipole responses inside a fluid-filled borehole could be different from the radiation pattern shown in this report. However, the amplitude variation with respect to formation velocities using the low-frequency and far-field approximation of the source radiation pattern agrees qualitatively with the observed amplitude behavior of sonic waveforms measured by DSI at the Mallik 5L-38 well.

P-wave amplitude loss due to source coupling is not limited to borehole sources. Appendix 1 illustrates the effect (determined in laboratory studies by Prasad and Dvorkin, 2004) of source coupling on the measured amplitude inside a brine; as indicated, about half the measured amplitude loss is due to source coupling.

\section{Calculation of Apparent Attenuation}

The amplitude variation shown in figure 1 can be interpreted from the intrinsic attenuation point of view, and this view has been adopted, for example, by Guerin and others (2005) and Matsushima (2005a). The amplitude can also be analyzed and interpreted as a consequence of the source radiation, defined here as the apparent attenuation to differentiate it from the intrinsic attenuation. The amplitude losses due to the intrinsic attenuation can be compared with those due to source coupling by using the apparent attenuation calibrated with the intrinsic attenuation at $j=1$.
Guerin and others (2005) used $12 \mathrm{kHz}$ and $2 \mathrm{kHz}$ for $\mathrm{P}$-wave and S-wave attenuation model studies, respectively, and these frequencies were used for the apparent attenuation in equations 10 and 11 . The distances from the monopole and dipole sources to receiver number 5 are approximately $3.5 \mathrm{~m}$ and $4.5 \mathrm{~m}$, respectively; these distances are used for equation 12.

Guerin and others (2005) derived the following relationships between the intrinsic attenuation and the gas hydrate saturation:

$$
\begin{gathered}
Q_{\mathrm{P}}^{-1}=0.0342+0.076 S_{\mathrm{h}}: \text { for monopole } \\
Q_{\mathrm{S}}^{-1}=0.0785+0.135 S_{\mathrm{h}}: \text { for upper dipole }
\end{gathered}
$$

where $S_{\mathrm{h}}$ is the gas hydrate saturation. In order to calibrate the apparent attenuation from the intrinsic attenuation, the intrinsic attenuation at $j=1$, or at depth of $843.84 \mathrm{~m}$, with $S_{\mathrm{h}}=0$ is used as the apparent attenuation at $j=1$. In other words, the apparent attenuations of P-waves $\left(Q_{\mathrm{aP}, 1}{ }^{-1}\right)$ and S-waves $\left(Q_{\mathrm{aS}, 1}{ }^{-1}\right)$ are 0.0342 and 0.0785 , respectively. Because the gas hydrate is present at depths greater than $891 \mathrm{~m}$ at the Mallik 5L-38 well, the intrinsic attenuations calculated by using equations 13a and $13 \mathrm{~b}$ are due to attenuation in water-saturated sediments, and these values are used to calibrate the amplitude loss due to the source coupling.

Figure $3 A$ shows the calculated apparent attenuation with respect to gas hydrate saturations estimated from the nuclear magnetic resonance tool (NMR) (Kleinberg and others, 2005). The calculated P-wave apparent attenuation is approximately one-half that of the intrinsic attenuation estimated by Guerin and others (2005), and the S-wave apparent attenuation appears to be comparable to the intrinsic attenuation.

If the source-coupling effect is used to account for the amplitude loss shown in figure $3 A$, the difference between the apparent attenuation and the attenuation measured by Guerin and others (2005), shown in figure $3 B$, can be interpreted as the intrinsic attenuation of GHBS.

\section{Discussion}

\section{Various Attenuation Measurements}

As mentioned previously, two opposing views exist for explaining the amplitude loss of the sonic waveform due to the presence of gas hydrate. One view is based on the sonic waveform analysis and suggests that the amplitude loss increases as gas hydrate saturation increases and is much greater than for non-gas-hydrate-bearing sediments (NGHBS). Arguments and evidence supporting intrinsic attenuation as the primary cause of the amplitude loss of the sonic waveform include the following: 

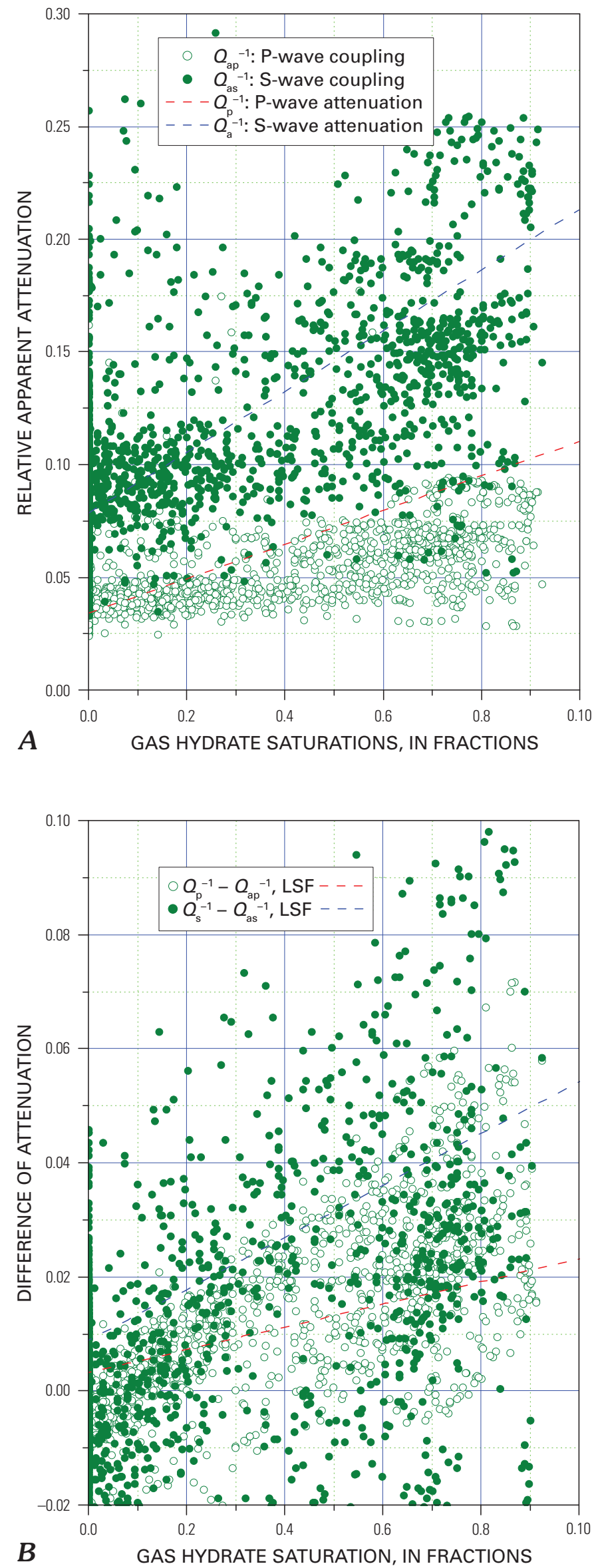

Figure 3. $A$, Intrinsic attenuation $\left(Q_{\mathrm{p}}^{-1}, Q_{\mathrm{s}}^{-1}\right)$ and apparent attenuation $\left(Q_{\mathrm{aP}}{ }^{-1}, Q_{\mathrm{as}}{ }^{-1}\right)$ with respect to gas hydrate saturations estimated from the nuclear magnetic resonance (NMR) tool at the Mallik $5 \mathrm{~L}-38$ well. The dashed lines are the least-square fits to the intrinsic attenuation estimated by Guerin and others (2005). $B$, The difference between the intrinsic attenuations estimated by Guerin and others (2005) and the apparent attenuations calculated by using the source coupling at the Mallik 5L-38 well.

1. From the analysis of hole-to-hole seismic surveys with frequencies ranging from 200 to 2,000 $\mathrm{Hz}$ at the Mallik well sites, Bauer and others (2005) demonstrated that there is frequencydependent $\mathrm{P}$-wave attenuation with a magnitude about one-half of that estimated from the DSI sonic $\operatorname{logs}(12 \mathrm{kHz}$ monopole and $2 \mathrm{kHz}$ dipole sources) by Guerin and others (2005). Compared to the waveform recorded at the source and receiver located in NGHBS, the high-frequency part of the waveform from formations highly saturated in gas hydrate decays more than the low-frequency part. The smaller attenuation in hole-to-hole seismic data compared to that estimated from the sonic waveforms is attributed to the lower frequency of the seismic source data compared to the sonic waveforms. Because the amplitude decay based on the lowfrequency approximation of the source radiation is independent of input frequency, it is not clear whether the source radiation can explain the observed frequency-dependent amplitude loss at the Mallik sites. In order to analyze the frequency-dependent amplitude loss of sonic waveforms by using the source radiation, numerical modeling techniques proposed by Tsang and Rader (1979), Cheng and Toksöz (1981), or Kurkjian and Chang (1986) could be used.

2. From the analysis of waveforms measured by using resonant-column experiments, Priest and others (2005) reported that P-wave attenuation increases with the increase of gas hydrate saturation. They interpreted the observed attenuation on the basis of an intercrack squirt flow. However, it should be noted that the P-wave attenuation is a maximum when the pores have approximately $3-5$ percent gas hydrate saturation and the $\mathrm{P}$-wave attenuation is higher than the $\mathrm{S}$-wave attenuation. Neither result agrees with observations at the Mallik sites.

3. Several authors have proposed various attenuation mechanisms to explain the amplitude loss in sonic waveforms measured for gas-hydratebearing sediments (GHBS): 
(a) Guerin and Goldberg (2005) applied the three-phase Biot equation proposed by Carcione and Tinivella (2000) with a squirt flow and friction between matrix and gas hydrate.

(b) Chand and Minshull (2004) applied the squirt flow mechanism proposed by Parra (2000) to interpret the sonic amplitude loss at the Mallik well sites.

(c) Dvorkin and Uden (2004) proposed that macroscopic squirt flow due to the heterogeneity in the formation is responsible for the large amplitude loss in GHBS.

Because causality requires a very specific relationship between velocity and attenuation, a large attenuation is generally associated with high velocity dispersion (Dvorkin and Uden, 2004). However, none of these three theories mentioned velocity or velocity dispersion except the theory of Guerin and Goldberg (2005), who discussed velocities only at particular frequencies. A problem with the Guerin and Goldberg (2005) approach is that at 100 percent gas hydrate saturation, the theory predicts the smallest P-wave attenuation (zero), but the highest $\mathrm{S}$-wave attenuation.

As shown in this report, some attenuation mechanisms have been proposed to explain the amplitude loss of the sonic waveform due to gas hydrate in the sediments, but none of the theories accurately predict both elastic wave velocities and amplitude losses for GHBS.

Arguments and evidence advanced for the opposing view that the amplitude loss for sonic waveforms measured in GHBS is less than for those in NGHBS include the following:

1. It is generally accepted that as sediments become more compacted or velocities become larger, the intrinsic attenuation becomes smaller. On the basis of this general perception, Gei and Carcione (2003) proposed that the attenuation in GHBS data is less than that in NGHBS data because gas hydrate, being stiffer than pore water, makes the porous medium more cohesive.

2. Intrinsic attenuation generally accompanies velocity dispersion. VSP data at the Mallik 2L-38 well indicate that the velocity dispersion at the Mallik site in the frequency range $20-12,000 \mathrm{~Hz}$ (surface seismic to sonic frequency) is negligible or very small (appendix 2). This result implies that the intrinsic attenuation of GHBS could be small.
3. The P-wave attenuation in GHBS, as estimated from the near-offset and far-offset VSP data, is less than that in NGHBS at the Mallik 3L-38 well (appendix 2). Also, Rossi and others (2005) demonstrated that the $\mathrm{P}$-wave attenuation of GHBS is less than that in NGHBS at offshore northwest Svalbard on the basis of the analysis of ocean-bottom seismograph data. Attenuation analysis of two VSPs at the Nankai Trough exploratory wells, offshore Japan, indicated no significant P-wave attenuation in GHBS, in contrast to high P-wave attenuations estimated from the sonic waveforms at the same wells (Matsushima, 2005b).

On the basis of data measured by varying the source environment (for example, well log, hole-to-hole seismic), it has been shown that amplitude loss increases as the gas hydrate saturation increases. However, data measured by holding a constant source environment (for example, surface VSP and marine ocean-bottom seismograph) indicate that amplitude loss decreases as gas hydrate saturation increases. These observations imply that the measured amplitude loss from a sonic waveform appears to be associated with source environments.

\section{Gas Hydrate Saturation and Attenuation}

The relationship between attenuation and gas hydrate saturation is given in equation 13, which indicates that the difference of attenuation at $S_{\mathrm{h}}=0$ percent and $S_{\mathrm{h}}=100$ percent is $\Delta Q_{\mathrm{P}}^{-1}=0.076$ for $\mathrm{P}$-waves and $\Delta Q_{\mathrm{S}}^{-1}=0.135$ for $\mathrm{S}$-waves. The least-squares fit to the difference of attenuation shown in figure $3 B$ is

$$
Q_{\mathrm{P}}^{-1}=0.003+0.02 S_{\mathrm{h}}: \text { for monopole }
$$

$$
Q_{\mathrm{S}}^{-1}=0.0009+0.04 S_{\mathrm{h}} \text { : for upper dipole. }
$$

In equations $14 \mathrm{a}$ and $14 \mathrm{~b}$, the intercepts of the linear function have no meaning because these are relative quantities, but the slopes do represent the attenuation dependence on hydrate saturation. Equations 14a and 14b yield the difference of attenuation at $S_{\mathrm{h}}=0$ percent and $S_{\mathrm{h}}=100$ percent, which is $\Delta Q_{\mathrm{P}}{ }^{-1}=0.02$ for $\mathrm{P}$-waves and $\Delta Q_{\mathrm{S}}{ }^{-1}=0.04$ for $\mathrm{S}$-waves. These values are three or four times smaller than those calculated by using equations $13 \mathrm{a}$ and $13 \mathrm{~b}$.

The difference of attenuation plotted in figure $3 B$ shows much scattering, so the estimates are not accurate. However, figures $3 A$ and $3 B$ suggest that the measured attenuation with hydrate saturation given by Guerin and others (2005) is possibly overestimated by at least a factor of two. 


\section{Intrinsic Attenuation and Source Coupling}

The objective of source-coupling analysis is determining whether the observed amplitude loss of sonic waveforms at the Mallik 5L-38 well site is primarily due to intrinsic attenuation or to source coupling. If source coupling is the dominant cause of the amplitude loss, the attenuation of the sonic waveforms measured for GHBS may not increase with increasing gas hydrate saturation. As discussed previously in this report, attenuation measurements using low-frequency sources with constant source environments support the view that the attenuation of the sonic waveforms measured for GHBS is less than that in NGHBS, whereas measurements using high-frequency sources with varying source environments support the opposite view. The results of source-coupling analysis still support the analysis of Guerin and others (2005), but the magnitude of intrinsic attenuation corrected for the source coupling is, at most, less than one-half that estimated by Guerin and others (2005).

The present study demonstrates that the amplitude variation observed at the Mallik 5L-38 well qualitatively agrees with that predicted by using source coupling. Furthermore, this study suggests that all analyses of amplitudes of sonic waveforms should include the effect of source coupling in order to accurately estimate the intrinsic attenuation of GHBS. The source-coupling formulation as presented here can be improved by (1) using high-frequency and near-field equations to model the source coupling, (2) including receiver coupling, and (3) analyzing frequency dependence of source and receiver coupling. These improvements could yield more accurate assessment of the intrinsic attenuation of GHBS.

If the measurements of the two opposing views relative to attenuations are both correct, the mechanism of attenuation in the high-frequency range may be different from that in the low-frequency range. In that case, the source coupling may only reduce the amount of attenuation, but may not change the slope of the plot in figure $3 B$. However, if it is assumed that the analysis of high-frequency measurement is erroneous owing to effects other than the intrinsic attenuation, other effects such as a source coupling should be investigated. The source coupling presented in this report could not have accounted for all the amplitude losses in the sonic waveforms because of approximated solutions. Although it is uncertain whether an improved formulation of the source coupling can resolve the controversy over the attenuation of sonic waveforms measured for GHBS, it is worth pursuing further, because there are evidencesalthough only in the low-frequency ranges-that high-frequency attenuation measurements in the borehole may be erroneous.

In order to reconcile the different interpretations of amplitude loss of sonic waveforms measured for GHBS and to investigate detailed mechanisms of attenuation of GHBS, more controlled experiments are required. As indicated in Hearst and others (2000), the dispersive nature of propagation modes due to the presence of a fluid-filled borehole makes data analysis of sonic waveforms complicated; one complication is the introduction of amplitude loss that is difficult to relate to the intrinsic attenuation of the formation.

\section{Conclusions}

This study demonstrates that the observed amplitude loss of sonic waveforms measured at the Mallik 5L-38 well is due to source coupling as well as to intrinsic attenuation of GHBS. The source coupling accounts for at least half of the observed amplitude loss due to gas hydrate at this well site. A consideration of the source coupling when analyzing a sonic waveform's amplitude loss indicates that both P-wave and $\mathrm{S}$-wave attenuations are not as high as previously estimated by Guerin and others (2005). The analysis presented herein emphasizes the importance of source coupling in analyzing waveforms that were acquired either by using borehole acoustic/seismic sources, such as monopole and dipole sources for the logging, or by using borehole sources for hole-to-hole seismic experiments.

The attenuation of GHBS is an important attribute to consider when attempting to identify and quantify in place gas hydrate through the use of seismic methods. Regardless of whether the attenuation increases or decreases with increasing gas hydrate saturation, the fact that such changes do occur significantly affects how the seismic data acquired for GHBS are interpreted. Data analysis of sonic waveforms is complicated by the dispersive nature of the propagation modes, which introduces amplitude loss that is difficult to relate to the intrinsic attenuation of the formation. Therefore, to fully understand the attenuation mechanism and to accurately measure the intrinsic attenuation of GHBS, controlled laboratory experiments are required.

\section{Acknowledgments}

Well logs at the Mallik 5L-38 gas hydrate research well and VSP data at the Mallik 3L-38 well were acquired by Schlumberger Ltd. The well was drilled to investigate gas hydrates in a collaborative research project among Japan National Oil Company, Japan Petroleum Exploration Company, Geological Survey of Canada, U.S. Geological Survey, GeoForschungsZentrum Potsdam, India Ministry of Petroleum and Gas, BP-ChevronTexaco-Burlington, U.S. Department of Energy, and the International Continental Scientific Drilling Program. I thank William Waite and John Miller for many discussions and helpful suggestions.

\section{References Cited}

Bauer, K., Haberland, C., Pratt, R.G., Hou, F., Medioli, B.E., and Weber, M.H., 2005, Ray-based cross-well tomography for P-wave velocity, anisotropy, and attenuation structure around the JAPEX/JNOC/GSC et al. Mallik 5L-38 gas hydrate production research well, in Dallimore, S.R., and Collett, T.S., eds., Scientific results from the Mallik 2002 gas hydrate production research well program, Mackenzie 
Delta, Northwest Territories, Canada: Geological Survey of Canada Bulletin 585, $21 \mathrm{p}$.

Carcione, J.M., and Tinivella, Umberta, 2000, Bottomsimulating reflectors-Seismic velocities and AVO effects: Geophysics, v. 65, p. 54-67.

Chand, Shyam, and Minshull, T.A., 2004, The effect of hydrate content on seismic attenuation-A case study for Mallik 2L-38 well data, Mackenzie Delta, Canada: Geophysical Research Letters, v. 31, L14609, doi: 10.1029/2004GL020292.

Cheng, C.H., and Toksöz, M.N., 1981, Elastic wave propagation in a fluid-filled borehole and synthetic acoustic logs: Geophysics, v. 46, p. 1,042-1,053.

Collett, T.S., Lewis, R.E., and Dallimore, S.R., 2005, JAPEX/ JNOC/GSC et al. Mallik 5L-38 gas hydrate production research well downhole well-log and core images, in Dallimore, S.R., and Collett, T.S., eds., Scientific results from the Mallik 2002 gas hydrate production research well program, Mackenzie Delta, Northwest Territories, Canada: Geological Survey of Canada Bulletin 585, 23 p.

Dvorkin, Jack, and Uden, Richard, 2004, Seismic wave attenuation in a methane hydrate reservoir: The Leading Edge, v. 23 , p. $730-734$.

Gei, Davide, and Carcione, J.M., 2003, Acoustic properties of sediments saturated with gas hydrate, free gas and water: Geophysical Prospecting, v. 51, p. 141-157.

Guerin, Gilles, and Goldberg, David, 2002, Sonic waveform attenuation in gas hydrate-bearing sediments from the JAPEX/JNOC/GSC Mallik 2L-38 gas hydrate research well, Mackenzie Delta, Northwest Territories, Canada: Journal of Geophysical Research, v. 107, no. B5, p. EPM1-1-EPM1-11.

Guerin, Gilles, and Goldberg, David, 2005, Modeling of acoustic wave dissipation in gas hydrate-bearing sediments: Geochemistry, Geophysics, Geosystems, v. 6, Q07010, doi: 10.1029/2005GC000918.

Guerin, Gilles, Goldberg, David, and Collett, T.S., 2005, Sonic attenuation in the JAPEX/JNOC/GSC et al. Mallik 5L-38 gas hydrate production research well, in Dallimore, S.R., and Collett, T.S., eds., Scientific results from the Mallik 2002 gas hydrate production research well program, Mackenzie Delta, Northwest Territories, Canada: Geological Survey of Canada Bulletin 585, 9 p.

Hearst, J.R., Nelson, P.H., and Paillett, F.L., 2000, Well logging for physical properties-A handbook for geophysicists, geologists and engineers: New York, John Wiley and Sons, $484 \mathrm{p}$.

Kleinberg, R.L., Flaum, C., and Collett, T.S., 2005, Magnetic resonance log of JAPEX/JNOC/GSC et al. Mallik 5L-38 gas hydrate production research well: Gas hydrate saturation, growth habit, and relative permeability, in Dallimore, S.R., and Collett, T.S., eds., Scientific results from the Mallik 2002 gas hydrate production research well program, Mackenzie Delta, Northwest Territories, Canada: Geological Survey of Canada Bulletin 585, 10 p.

Kurkjian, A.L., and Chang, S.K., 1986, Acoustic multiple sources in fluid-filled boreholes: Geophysics, v. 51, p. $148-163$.

Lee, M.W., 1986, Low-frequency radiation for point sources in a fluid-filled borehole: Geophysics, v. 51, p. 1,801-1,807.

Lee, M.W., 1990, Traveltime inversion using transmitted wave of offset VSP data: Geophysics, v. 55, p. 1,089-1,097.

Lee, M.W., and Balch, A.H., 1982, Theoretical seismic wave radiation from a fluid-filled borehole: Geophysics, v. 47, p. $1,308-1,314$.

Matsushima, Jun, 2005a, Attenuation measurement from sonic waveform logs in methane hydrate-bearing sediments at the Nankai Trough exploratory well off Tokai, central Japan: Geophysical Research Letters, v. 32, L03306, doi: 10.1029/2004GL021786.

Matsushima, Jun, 2005b, Seismic wave attenuation in methane hydrate-bearing sediments-Vertical seismic profiling data from the Nankai Trough exploratory well, offshore Tokai, central Japan: Eos (Transactions, American Geophysical Union), v. 86, no. 52, Fall Meeting Supplement, Abstract MR41A-0908.

Milkereit, Bernd, Adam, E., Li, Z., Qian, W., Bohlen, T., Banerjee, D., and Schmitt, D.R., 2005, Multi-offset vertical seismic profiling-An experiment to assess petrophysicalscale parameters at the JAPEX/JNOC/GSC et al. Mallik 5L-38 gas hydrate production research well, in Dallimore, S.R., and Collett, T.S., eds., Scientific results from the Mallik 2002 gas hydrate production research well program, Mackenzie Delta, Northwest Territories, Canada: Geological Survey of Canada Bulletin 585, 13 p.

Parra, J.O., 2000, Poroelastic model to relate seismic wave attenuation and dispersion to permeability anisotropy: Geophysics, v. 65, no. 1, p. 202-210.

Prasad, Manika, and Dvorkin, Jack, 2004, Velocity and attenuation of compressional waves in brines: Society of Exploration Geophysicists Technical Program Expanded Abstracts, p. 1,666-1,669, doi: 10.1190/1.1845150

Priest, Jeffrey, Best, Angus, Clayton, Chris, and Watson, Emily, 2005, Seismic properties of methane gas hydratebearing sands: Proceedings of the Fifth International Conference on Gas Hydrate, June 13-16, Trondheim, Norway, v. 2, p. $440-447$.

Rossi, Giuliana, Madrussani, Gianni, Gei, Davide, Böhm, Gualtiero, and Camerlenghi, Angelo, 2005, Velocity and 
attenuation 3D tomography for gas-hydrates studies-The NW offshore Svalbard case: Proceedings of the Fifth International Conference on Gas Hydrate, June 13-16, Trondheim, Norway, v. 2, p. 677-682.

Schön, J.H., 1998, Physical properties of rocks: Oxford, Pergamon, $583 \mathrm{p}$.

Tang, X.M., Dubinsky, Vladimir, Wang, Tsili, Bolshakov, Alexei, and Patterson, Doug, 2002, Shear-velocity measurement in the logging-while-drilling environment-
Modeling and field evaluations: Transactions of the SPWLA 43rd Annual Logging Symposium, Oiso, Japan, June 2-5, paper RR, $14 \mathrm{p}$.

Tolstoy, Ivan, and Clay, C.S., 1966, Ocean acoustics: New York, McGraw-Hill, 293 p.

Tsang, Leung, and Rader, Dennis, 1979, Numerical evaluation of transient acoustic waveforms due to a point source in a fluid-filled borehole: Geophysics, v. 44, p. 1,706-1,720. 


\section{Appendix 1. Amplitude Loss of Compressional Waves in Brine Due to Source Coupling}

To understand the elastic properties of frozen sediments and to get some insight into the attenuation mechanism of gas-hydrate-bearing sediments, Prasad and Dvorkin (2004) performed pulse transmission experiments in brine for temperatures ranging from $18^{\circ}$ to $-21^{\circ} \mathrm{C}$. Appendix 2 analyzes the amplitude loss of unfrozen brine (temperatures from $18^{\circ}$ to $-3^{\circ} \mathrm{C}$ ) in order to assess the source-coupling effect on the amplitude of P-waves.

The radiation of a $\mathrm{P}$-wave from a point source in unbounded water $\left(U_{\mathrm{p}}\right)$ can be written as (Tolstoy and Clay, 1966)

$$
U_{\mathrm{P}} \propto \sqrt{\frac{\alpha_{\mathrm{w}}}{\rho_{\mathrm{w}}}}
$$

where $\rho_{\mathrm{w}}$ and $\alpha_{\mathrm{w}}$ are the density and P-wave velocity of water, respectively. As temperature increases, the density of brine decreases and velocity increases.

Figure 1-1 shows the normalized measured amplitude and normalized amplitude variation due to source coupling to the brine. In this calculation, measured velocities by Prasad and Dvorkin (2004) and calculated densities accounting for temperature variation were used. Figure 1-1 indicates that about half of the measured amplitude loss is due to source coupling.

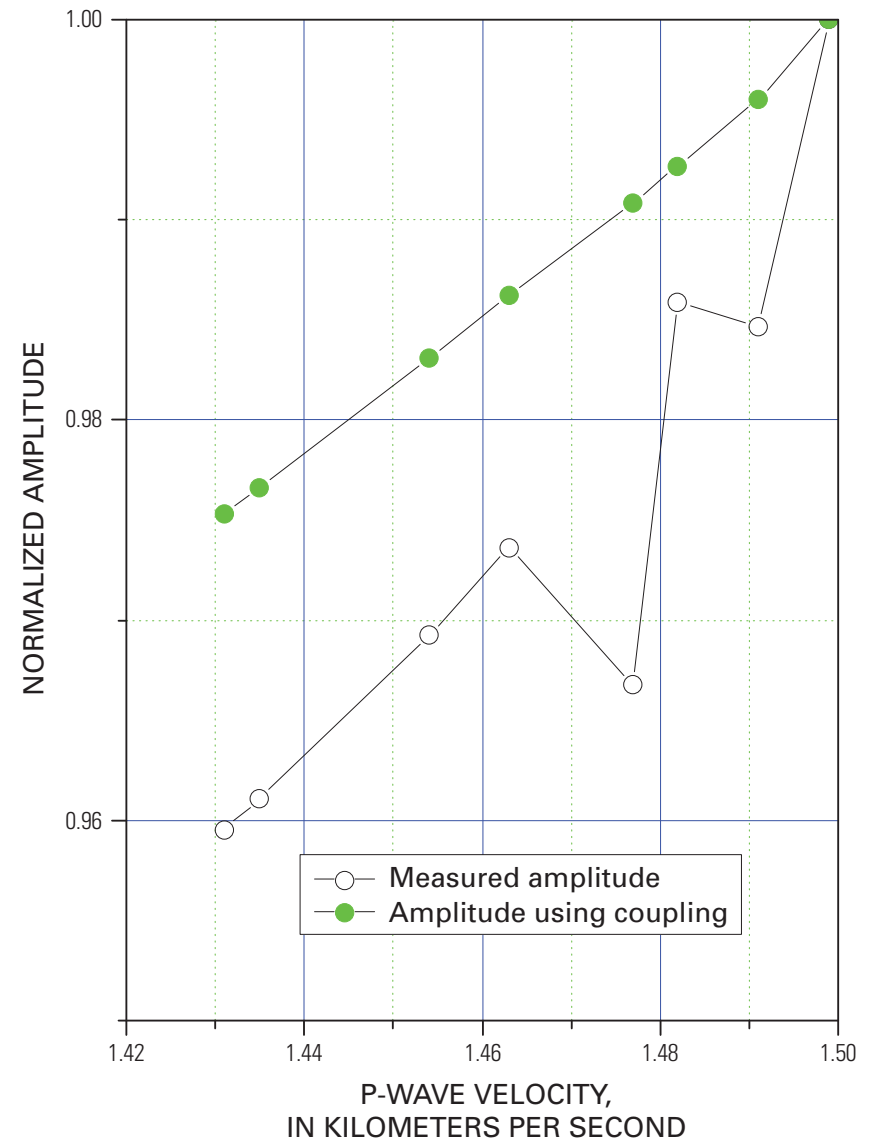

Figure 1-1. P-wave amplitude loss due to source coupling is compared to the amplitude loss inside brine measured by Prasad and Dvorkin (2004). 


\section{Appendix 2. Attenuation at Mallik 3L-38}

Attenuation is assumed to be proportional to the first power of frequency (Schön, 1998) and is given by

$$
A_{j}(f)=H A_{0}(f) \exp \left(-\lambda_{j} f R_{j}\right) \equiv H A_{0}(f) \exp \left(-\alpha_{j} R_{j}\right),
$$

where $A_{j}(f)$ is the amplitude spectrum at distance $R_{i}, f$ is the linear frequency, $\alpha_{j}$ is the attenuation coefficient $\left(\alpha_{j}=\lambda_{j} f\right), A_{0}$ is the input or reference amplitude spectrum, and $H$ is a constant representing frequency-independent amplitude loss such as loss due to the geometrical spreading.

If a constant-input wavelet is assumed, the attenuation coefficient can be computed with the following equation:

$$
\ln \left(\frac{A_{j}(f)}{A_{0}(f)}\right)=\ln H-\lambda_{j} f R_{j} \equiv a-b f
$$

where $a$ and $b$ are the intercept and the slope, respectively, of the least-squares fit of the amplitude ratio.

The quality factor $(Q)$, which is the inverse of attenuation, is calculated as (Schön, 1998) follows:

$$
Q=\frac{\pi f}{\alpha V}=\frac{\pi R}{b V}
$$

where $V$ is the interval velocity.

Figure 2-1 shows the spectra ratios of A875 to A560 (a line connected with open circles) and A1115 to A560 (a line connected with dots), where A560, A875, and A1115 are the amplitude spectra at depths of $560 \mathrm{~m}, 875 \mathrm{~m}$, and $1,115 \mathrm{~m}$, respectively. Sediments at depths between $560 \mathrm{~m}$ and 875 $\mathrm{m}$ are water-saturated sediments or non-gas-hydrate-bearing sediments (NGHBS), and sediments at depths between 875 $\mathrm{m}$ and $1,115 \mathrm{~m}$ are mostly gas-hydrate-bearing sediments (GHBS) (actually $890 \mathrm{~m}$ and 1,109 $\mathrm{m}$ are the exact top and bottom of GHBS at Mallik 3L-38).

By using an average velocity of $2.07 \mathrm{~km} / \mathrm{s}$ and equation 2-2, with $b_{1}=6 \times 10^{-3}$ and $R=315 \mathrm{~m}$, the quality factor determined for NGHBS is 80 . The attenuation between $875 \mathrm{~m}$ and $1,115 \mathrm{~m}$ can be calculated with the spectra ratios shown in figure 2-1. By using the average velocity of $2.54 \mathrm{~km} / \mathrm{s}, R=$ $240 \mathrm{~m}$, and $b=1.9 \times 10^{-3}$, equation $2-1$ yields the coefficient of $b$ for GHBS as $b=b_{2}-b_{1}$, and the quality factor of GHBS is 156 . Thus, the attenuation $\left(Q^{-1}\right)$ of NGHBS $(0.0125)$ is higher than that of GHBS (0.0064).

\section{Traveltime Inversion}

If a layered medium is assumed, the first arrival times of two offset VSP data can be used to derive interval velocities and dip angles (Lee, 1990). The arrival times and offsets used in the present study are from Milkereit and others (2005). Vertical components of the zero-offset and offset P-wave VSP

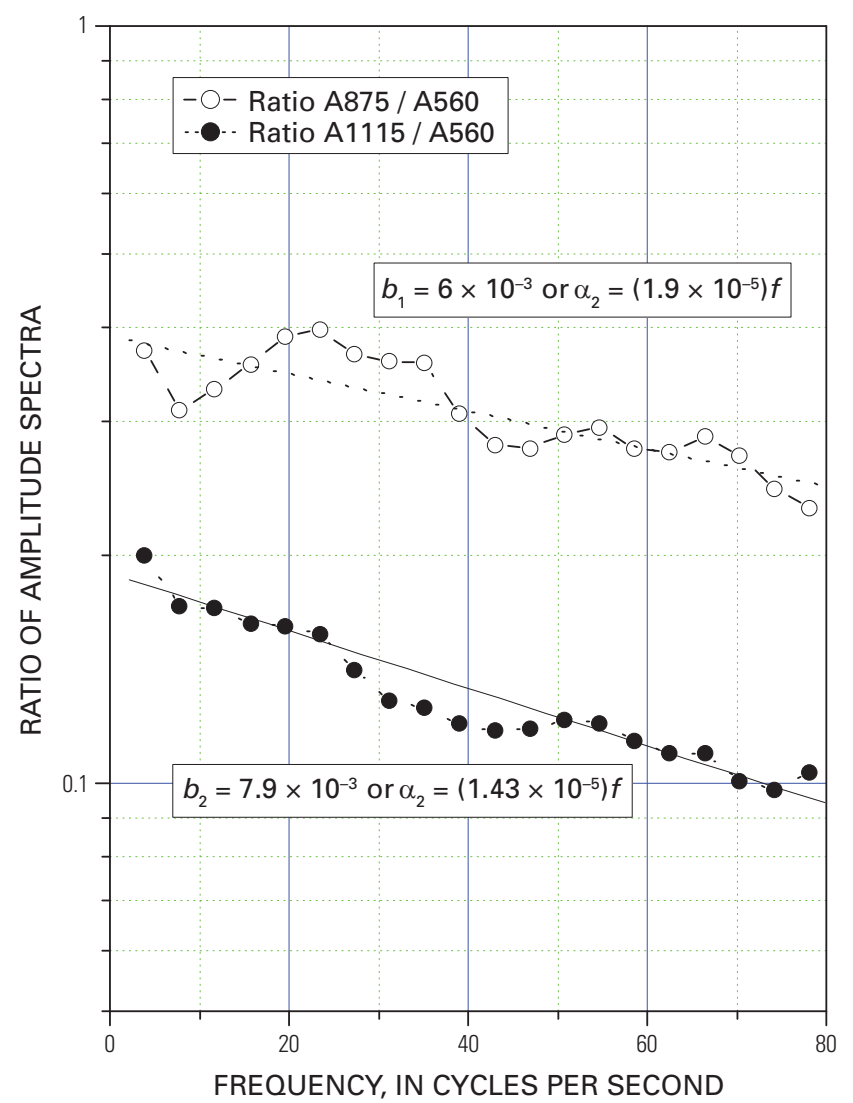

Figure 2-1. Spectral ratios at the Mallik $3 \mathrm{~L}-58$ well, $45 \mathrm{~m}$ southwest of Mallik 5L-38 well. A560, A875, and A1115 are amplitude spectra at the depths of $560 \mathrm{~m}, 875 \mathrm{~m}$, and 1,115 m, respectively.

data were used to derive P-wave velocity, assuming that the dip angles are negligible.

As shown in figure 2-2, estimated P-wave interval velocities from the near-offset VSP data (offset $=-22 \mathrm{~m}$ ) are $3.26,2.09$, and $2.56 \mathrm{~km} / \mathrm{s}$ for the permafrost, NGHBS, and GHBS, respectively. Figure 2-2 also shows the interval velocities derived from the traveltime inversion by using the three offset VSP data (-22-, 83-, and 257-m offsets) and by using sonic log data at the Mallik 5L-38 well. The general trend of the interval velocities from the offset VSP data agrees well with that from the sonic log. The average velocity for GHBS derived from the offset VSP data is $2.56 \mathrm{~km} / \mathrm{s}$, which is the same as the velocity derived from the near-offset data, whereas the average velocity is $2.66 \mathrm{~km} / \mathrm{s}$ for the sonic $\log$ at the Mallik 5L-38 well. The P-wave velocity from the sonic $\log$ is about 4 percent faster than that derived from the VSP data. Although velocity dispersion is assumed to cause the discrepancy between the sonic log and VSP results, the velocity dispersion in this case is small. Heterogeneity within the medium may cause the disagreement, but this is not certain. 


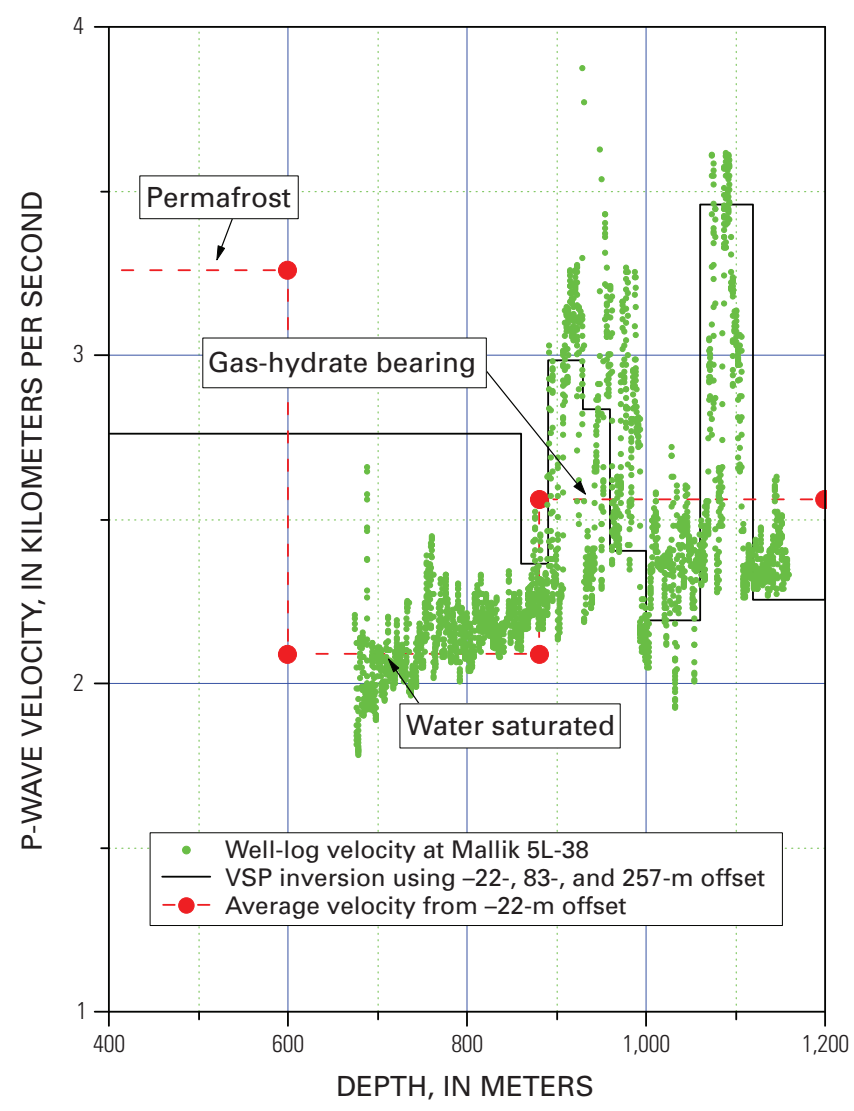

Figure 2-2. A graph showing velocities derived from the VSP data at the Mallik $3 \mathrm{~L}-58$ well and well-log velocities measured at the Mallik 5L-38. 